\title{
The Pennsylvania State University College of Engineering
}

\section{Final Report \\ Analysis for Thermo-Chemical Decomposition of Composite Structures}

N.J. Salamon, Principal Investigator

NASA Grant NAG8-879

Period: June 1993 - March 1995

Mark Stucker, NASA Technical Officer

Marshall Space Flight Center

Department of Engineering Science and Mechanics

227 Hammond Building

University Park, PA 16802 


\section{Note to readers}

This report is the manuscript for a paper to soon appear in the AIAA J. of Thermophysics and Heat Transfer. The delay in its appearance after completion of the research is because it was first submitted to an applied mechanics journal where it was accepted subject to significant reduction in content. It was withdrawn from that journal because such a reduction would detract from its usefulness. 


\title{
Finite Element Analysis of Poroelastic Composites Undergoing Thermal and Gas Diffusion
}

\author{
Sunpyo Lee (Full Time Lecturer) \\ Department of Mechanical Engineering, Kyonggi University \\ Suwon, Korea 440-760 \\ Nicholas J. Salamon (Professor) \\ Department of Engineering Science and Mechanics \\ The Pennsylvania State University \\ 227 Hammond Building, University Park, PA 16802 \\ and \\ Roy M. Sullivan (Aerospace Engineer) \\ Structures and Dynamics Laboratory \\ George C. Marshall Space Flight Center \\ Marshall Space Flight Center, AL 35812
}

\begin{abstract}
A theory for time-dependent thermal and gas diffusion in mechanically time-rateindependent anisotropic poroelastic composites has been developed. This theory advances previous work by the latter two authors by providing for critical transverse shear through a three-dimensional axisymmetric formulation and using it in a new hypothesis for determining the Biot fluid pressure-solid stress coupling factor. The derived governing equations couple material deformation with temperature and internal pore pressure and more strongly couple gas diffusion and heat transfer than the previous theory. Hence the theory accounts for the interactions between conductive heat transfer in the porous body and convective heat carried by the mass flux through the pores. The Bubnov Galerkin finite element method is applied to the governing equations to transform them into a semidiscrete finite element system. A numerical procedure is developed to solve the coupled equations in the space and time domains. The method is used to simulate two high temperature tests involving thermal-chemical decomposition of carbon-phenolic composites. In comparison with measured data, the results are accurate. Moreover unlike previous work, for a single set of poroelastic parameters, they are consistent with two measurements in a restrained thermal growth test.
\end{abstract}




\section{Nomenclature}

$c_{1}$ : degree of processing

$C_{\mathrm{p}}$ : heat capacity

$e_{\mathrm{i}}$ : elastic strains

$e_{i}^{\text {tot: total strains }}$

$h_{\mathrm{g}}$ : heat enthalpy

$h_{\mathrm{R}}:$ heat of reaction

$K_{\mathrm{g}}$ : gas bulk modulus

k: permeability matrix

$M$ : Biot's material constant

$M W_{\mathrm{g}}:$ molecular weight

$m_{\mathrm{g}}$ : gas mass increment per unit bulk volume

$N_{\mathrm{i}}$ : elemental shape functions

$p:$ pore pressure

q: heat flux vector

$R$ : universal gas constant

$T$ : absolute temperature

$u, w$ : radial and axial displacement components

$\mathbf{v}_{\mathrm{g}}$ : average gas velocity

$\alpha_{\mathrm{i}}$ : Biot's material constants; pressure-stress coupling factors

$\beta_{\mathrm{i}}$ : thermal expansion coefficients of solid

$\beta_{\mathrm{g}}$ : thermal expansion coefficient of gas

$\delta_{\mathrm{i}}$ : unjacketed compressibilities

$\phi$ : porosity

$\kappa:$ thermal conductivity matrix

$\mu:$ gas viscosity

$\rho_{\mathrm{g}}:$ gas density

$\rho_{\text {proc }}$ : density of processed solid 
$\rho_{\mathrm{s}}:$ solid density

$\rho_{\text {virg }}$ : density of virgin solid

$\tau_{i}:$ total stresses

$\zeta$ : gas volume increment per unit bulk volume

$\zeta^{\text {tot}: ~ t o t a l ~ g a s ~ v o l u m e ~ i n c r e m e n t ~ p e r ~ u n i t ~ b u l k ~ v o l u m e ~}$ 


\section{INTRODUCTION}

Poroelasticity has been applied to numerous problems in which a fluid, diffusing through a deformable solid, influences the mechanical behavior in a coupled manner. The most common applications involving solid deformation are in geotechnical engineering [1]. Problems coupling fluid diffusion to thermal or electronic diffusion have also been solved within a rigid matrix. Such problems feature two independent field variables, namely temperature and pressure. Raising the level of complexity to three or more independent field variables, material deformation has been coupled to gas and thermal diffusion in the study of a high temperature thermal insulation material by Sullivan [2], Sullivan and Salamon [3], from which this work is launched, and Weiler[4].

The theory is founded upon that of Biot [5] who developed constitutive relations for the elastic behavior of saturated, isotropic porous soils and Biot and Willis [6] who expanded them to include anisotropy. Their theory provides a mathematical model for the mechanical behavior of the bulk material by phenomenologically linking the interaction between the solid and fluid phases in the sense of "mixtures". Nur and Byerlee [7] discussed the concept of effective stress and defined an effective stress law for isotropic, fluid-filled porous materials. Carroll [8] further developed the effective stress law for anisotropic porous materials and Kurashige [9] reported an anisotropic, thermoelastic formulation. All these formulations are for treatment of geo-materials.

The class of problems treated here involve an evolutionary process in time during which solid deformation, pore pressure (due to diffusing gases) and change in temperature in poroelastic bodies interact. In general the process may involve fluid mass generation associated with conversion of a solid from a virgin state to a processed state driven by a change in energy level in a control volume through which mass and energy flux may transfer. In particular, the present application deals with thermally activated, chemical decomposition of carbon-phenolic composite material used in rocket liners. The theory is more general and may have other applications.

Carbon-phenolic is a polymeric material which chemically decomposes into solid carbon (char) and pyrolysis gases when exposed to high temperature. Initiating at an exposed surface, a char layer forms, governed by chemical reaction kinetics, and advances 
into the material lagging the thermal front. The solid carbon left behind has a porous structure which permits generated process or decomposition gases to escape. This convective action assisted by a low thermal conductivity of char insulates the interior virgin material from heat. Hence carbon-phenolic is suitable for high temperature insulation applications.

This paper advances the theory in $[2,3]$ by (1) creating a model for nonuniform, thermal convective fields, (2) developing the coupled, three-dimensional, axisymmetric finite element formulation for anisotropic, axisymmetric structures in a general form and (3) employing a new empirical, but physical form for Biot's pressure-stress coupling factor. It then uses this formulation to simulate two high temperature tests done by Stokes [10] and, after determining a consistent set of poroelastic parameters, achieves excellent agreement with the test data. The following sections cover the theory and finite element formulation, both contain new approaches, and then apply them to experiments on decomposing carbon phenolic specimens.

\section{GENERAL THEORY}

The problem is first formulated in a Cartesian frame and then set into cylindrical coordinates $(r, \theta, z)$ such that $r$ is the radius of a cylinder and $z$ is the generator normal to the $r, \theta$-plane where $\theta$ is the angle of rotation about $z$. Axial symmetry is invoked by subjecting all fields $(\cdot)$ to the constraint $\partial(\cdot) / \partial \theta=0$. The resultant degrees of freedom are displacements $u, w$ in the $r, z$ directions respectively, pore pressure $p$ and temperature $T$.

Material points are occupied by both solid and fluid, overlaid in the sense of mixtures, and constitute the bulk material. The porous solid is material devoid of fluid. Moreover solid and fluid refer to pure solid and pure fluid material, respectively. Stresses are usually referenced to bulk area, hence they are termed effective. However any quantity

denoted $(\cdot)^{\mathrm{S}}$ pertains to the pure solid; such stresses are referenced to pure solid area. Pressure $p$ is that in the pure fluid, i.e., herein the gas.

Mass flux through the porous solid is assumed to be slow, irrotational flow. Temperature at a material point is assumed common to both the solid and fluid, hence perfect heat transfer occurs between them in a control volume. Heat transfer occurs as conduction 
in the solid and as convection by diffusion of the gas.

The theory is formulated to achieve a linear, time-marching solution. Nonlinear expressions which arise are linearized.

\subsection{Constitutive Equations}

We consider a linear elastic material with porosity $\phi$ and stiffness $C$ whose pores are saturated with gas under pressure $p$ which is taken positive for compression. The small elastic strain in the solid, in terms of total and thermal strains, is $e_{\mathrm{i}}=e_{\mathrm{i}}^{\text {tot }}-\beta_{\mathrm{i}} \Delta T$. Similarly the elastic pore fluid volume fraction change is $\zeta=\zeta^{\text {tot }}+\phi \beta_{\mathrm{g}} \Delta T$ with a plus sign because pore fluid is lost under a temperature increase at constant pressure. The semi-complementary form of strain energy $W[11]$ is

$$
W=\frac{1}{2}\left(C_{i j} e_{i} e_{j}-2 \alpha_{i} e_{i} p-\frac{1}{M} p^{2}\right)
$$

from which the total stresses $\tau_{\mathrm{i}}$ (solid plus fluid pressure referenced to the bulk area) and total fluid volume increment per unit bulk volume $\zeta^{\text {tot }}$ follow:

$$
\begin{gathered}
\tau_{i}=\frac{\partial W}{\partial e_{i}}=C_{i j}\left(e_{j}^{t o t}-\beta_{j} \Delta T\right)-\alpha_{i} p \\
\zeta^{t o t}=-\frac{\partial W}{\partial p}-\phi \beta_{8} \Delta T=\frac{1}{M} p+\alpha_{i}\left(e_{i}^{t o t}-\beta_{i} \Delta T\right)-\phi \beta_{g} \Delta T
\end{gathered}
$$

where repeated subscripts are summed. Reduced indicial notation, employed to represent tensorial quantities, will be defined subsequently.

In equation (3), the coefficient $M$ is determined through the isothermal unjacketed test, Biot and Willis [6], and can be expressed in terms of Biot's coefficients $\alpha_{i}$ and unjacketed compressibilities $\delta_{\mathrm{i}}$ as

$$
M=\frac{K_{g}}{\phi-\phi\left(\delta_{1}+\delta_{2}+\delta_{3}\right) K_{g}+\alpha_{i} \delta_{i} K_{g}}
$$


where $K_{\mathrm{g}}=-V(\partial p / \partial V)_{\mathrm{T}}$ is the gas bulk modulus at constant temperature taken positive for decreasing volume under compression. For an ideal gas, $K_{\mathrm{g}} \sim p$ and the last term in equation (3) varies as $\Delta T / T$ which at high temperatures will be small and may be ignored.

The compressibilities may be determined directly from the unjacketed test, or by referring to Carroll [8],

$$
\delta_{i}=\sum_{m=1}^{3} S_{i m}^{s}
$$

where $S^{S}$ denotes an elastic compliance of the solid phase material. An alternative method is to first set $\alpha_{i}$, then use (see the dissertation by Lee [12])

$$
\left\{\delta_{i}\right\}=\left[C_{i j}\right]^{-1}\left(\{1\}-\left\{\alpha_{i}\right\}\right)
$$

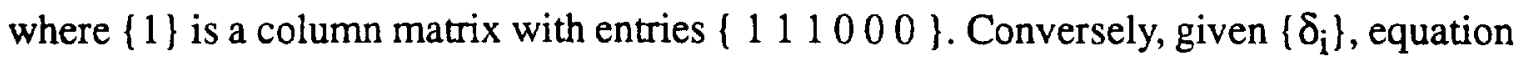
(6) may be inverted to determine $\left(\alpha_{i}\right\}$. The alternative route is adopted here because the freedom to determine $\alpha$ from experiment counters uncertainty in the values of the material constants during decomposition.

For further information, details are in [12]. A general development in Cartesian coordinates is given by Weiler [4]; an alternative is given by Kurashige [9].

Axisymmetric considerations. The most general material which meets the axisymmetric constraint is monoclinic with material symmetry about the $r, z$-plane [12] and is referred to here as $r, z$-symmetric. The elastic stiffness matrix for this material is written (using the notation $1 \equiv r r, 2 \equiv \theta \theta, 3 \equiv z z, 4 \equiv \theta z, 5 \equiv r z$ and $6 \equiv r \theta$ ) as

$$
[C]=\left[\begin{array}{cccccc}
C_{11} & C_{12} & C_{13} & 0 & C & 0 \\
& C_{22} & C_{23} & 0 & C & 0 \\
& & C_{33} & 0 & C & 0 \\
& & & C_{44} & 0 & C_{46} \\
& & & & C_{55} & 0 \\
\text { Sym } & & & & & C_{66}
\end{array}\right]
$$


and the material has $\alpha_{4}=\alpha_{6}=0$ and $\beta_{4}=\beta_{6}=0$. For this case, the constitutive equation (2) becomes

$$
\left\{\begin{array}{c}
\tau_{1} \\
\tau_{2} \\
\tau_{3} \\
\tau_{4} \\
\tau_{5} \\
\tau_{6}
\end{array}\right\}=[C]\left\{\begin{array}{c}
e_{1}^{t o t}-\beta T \\
e_{2}^{t o t}-\beta T \\
e_{3}^{t o t}-\beta T \\
e_{4}^{t o t} \\
e_{5}^{t o t}-\beta T \\
e_{6}^{t o t}
\end{array}\right\}-p\left\{\begin{array}{c}
\alpha_{1} \\
\alpha_{2} \\
\alpha_{3} \\
0 \\
\alpha_{5} \\
0
\end{array}\right\}
$$

where

$e_{1}^{t o t}=\frac{\partial u}{\partial r} \quad e_{2}^{t o t}=\frac{u}{r} \quad e_{3}^{t o t}=\frac{\partial w}{\partial z} \quad e_{5}^{t o t}=\frac{\partial u}{\partial z}+\frac{\partial w}{\partial r} \quad e_{4}^{t o t}=e_{6}^{t o t}=0$

\subsection{Momentum Equations}

In cylindrical coordinates $(r, \theta, z)$, the equations which govern the motion of the porous, fluid-filled material due to external loads in the absence of body forces and under quasi-static conditions are written as

$$
\begin{gathered}
\frac{\partial \tau_{1}}{\partial r}+\frac{1}{r} \frac{\partial \tau_{6}}{\partial \theta}+\frac{\partial \tau_{5}}{\partial z}+\frac{1}{r}\left(\tau_{1}-\tau_{2}\right)=0 \\
\frac{\partial \tau_{6}}{\partial r}+\frac{1}{r} \frac{\partial \tau_{2}}{\partial \theta}+\frac{\partial \tau_{4}}{\partial z}+\frac{1}{r}\left(\tau_{6}+\tau_{4}\right)=0 \\
\frac{\partial \tau_{5}}{\partial r}+\frac{1}{r} \frac{\partial \tau_{4}}{\partial \theta}+\frac{\partial \tau_{3}}{\partial z}+\frac{1}{r} \tau_{5}=0
\end{gathered}
$$

Axisymmetric case. Under the constraints of axisymmetric conditions $(v=0$ and $\partial(\cdot) / \partial \theta=0), e_{4}{ }^{\mathrm{tot}}=e_{6}{ }^{\mathrm{tot}}=0$ from equation $(9), \tau_{4}=\tau_{6}=0$ from equation (8) and the sec- 
ond equation in (10) is automatically satisfied. Then the equations reduce to

$$
\begin{gathered}
\frac{\partial \tau_{1}}{\partial r}+\frac{\partial \tau_{5}}{\partial z}+\frac{1}{r}\left(\tau_{1}-\tau_{2}\right)=0 \\
\frac{\partial \tau_{5}}{\partial r}+\frac{\partial \tau_{3}}{\partial z}+\frac{1}{r} \tau_{5}=0
\end{gathered}
$$

\subsection{Gas Diffusion Equation}

The differential equation governing the flow of fluid through the anisotropic porous solid skeleton, derived from the principle of conservation of mass within a control volume, is

$$
\frac{\partial m_{g}}{\partial t}+\nabla \cdot\left(\rho_{g} v_{g}\right)=\frac{\partial m_{g}^{g e n}}{\partial t}
$$

where $\rho_{\mathrm{g}}$ and $v_{\mathrm{g}}$ are the mass density and velocity of the gas, respectively, and $m_{\mathrm{g}}{ }^{\text {gen }}>0$ is the mass generation term $\left(m_{\mathrm{g}}{ }^{\text {gen }}<0\right.$ is the mass consumed). On the left hand side, the first term is the storage term which represents the time rate of gas mass inside the control volume and the second term is the diffusion term which defines the flux of gas mass crossing the boundaries of the control volume.

We assume the gas density $\rho_{\mathrm{g}}$ constant during an incremental change in time.

Hence it is treated as a parameter which is updated at each numerical time step. The incremental change in gas mass per unit bulk volume is obtained by multiplying the total volume increment per unit bulk volume $\zeta^{\text {tot }}$ by the gas density $\rho_{\mathrm{g}}$. Therefore

$$
\frac{\partial m_{g}}{\partial t}=\rho_{g} \frac{\partial \zeta^{t o t}}{\partial t}
$$

Hence from equation (3), the mass storage term is written in terms of pressure, strains and temperature as 


$$
\frac{\partial m_{g}}{\partial t}=\rho_{g}\left[\frac{1}{M} \frac{\partial p}{\partial t}+\alpha_{i} \frac{\partial e_{i}^{t o t}}{\partial t}-\left(\alpha_{i} \beta_{i}+\phi \beta_{g}\right) \frac{\partial T}{\partial t}\right]
$$

In the second term on the left hand side of equation (12), the volume average gas velocity components in cylindrical coordinates are expressed by Darcy's law for slow, irrotational flow as

$$
v_{g}=-\frac{1}{\mu} \mathbf{k} \cdot \nabla p
$$

where $\mu$ is the gas viscosity and

$$
\mathbf{k}=\left[\begin{array}{lll}
k_{1} & k_{6} & k_{5} \\
k_{6} & k_{2} & k_{4} \\
k_{5} & k_{4} & k_{3}
\end{array}\right]
$$

is the permeability matrix for an anisotropic porous body.

The right hand side of equation (12) represents the rate of increase in gas mass per unit bulk volume due to some chemical conversion or phase change process and this is equal to the rate at which the solid phase gives up or takes in mass per unit bulk volume. Mathematically,

$$
\frac{\partial m_{g}^{g e n}}{\partial t}=-\frac{\partial \rho_{s}}{\partial t}
$$

where the solid density $\rho_{s}$ may be expressed as a linear function of the degree of processing $c_{1}$ as in [13], hence

$$
\rho_{s}=c_{1} \rho_{\text {virg }}+\left(1-c_{1}\right) \rho_{\text {proc }}
$$

in which $c_{1}$ is unity at the virgin stage and zero at the completely processed stage. Expanding equation (17) using the chain rule,

$$
\frac{\partial m_{g}^{g e n}}{\partial t}=-\frac{d \rho_{s}}{d c_{1}} \frac{d c_{1}}{d t}=-\left(\rho_{v i r g}-\rho_{p r o c}\right) \frac{d c_{1}}{d t}
$$


in which the rate of processing $d c_{1} / d t$ is defined in Section 4.1 by setting up a series of Arrhenius kinetic reaction equations based on experimental data.

Substituting equation (14), (15), (16) and (19) into equation (12) gives

$$
\begin{aligned}
& \frac{\rho_{g}}{M}\left(\frac{\partial p}{\partial t}\right)+\rho_{g} \alpha_{i}\left(\frac{\partial e_{i}^{t o t}}{\partial t}\right)-\rho_{g}\left(\alpha_{i} \beta_{i}+\phi \beta_{g}\right)\left(\frac{\partial T}{\partial t}\right) \\
& +\left(\rho_{v i r g}-\rho_{\text {proc }}\right)\left(\frac{d c_{1}}{d t}\right)-\rho_{g} \nabla \cdot\left(\frac{1}{\mu} \mathbf{k} \cdot \nabla p\right)=0
\end{aligned}
$$

where gas density is treated as a constant locally in space. Equation (20) involves temporal and spatial derivatives of the gas pore pressure and accounts for the changes in pore pressure due to chemical processing, temperature variations and solid deformations.

Axisymmetric case. The gas diffusion equation (20) is reduced for a monoclinic $r$, $z$-symmetric material in cylindrical coordinates to

$$
\begin{gathered}
\frac{\rho_{g}}{M}\left(\frac{\partial p}{\partial t}\right)+\rho_{g} \frac{\partial}{\partial t}\left[\alpha_{1} \frac{\partial u}{\partial r}+\alpha_{2} \frac{u}{r}+\alpha_{3} \frac{\partial w}{\partial z}+\alpha_{5}\left(\frac{\partial w}{\partial r}+\frac{\partial u}{\partial z}\right)\right] \\
-\rho_{g}\left(\alpha_{1} \beta_{1}+\alpha_{2} \beta_{2}+\alpha_{3} \beta_{3}+\alpha_{5} \beta_{5}+\phi \beta_{g}\right) \frac{\partial T}{\partial t}+\left(\rho_{v i r g}-\rho_{\text {proc }}\right) \frac{d c_{1}}{d t} \\
-\rho_{g}\left\{\frac{1}{r} \frac{\partial}{\partial r}\left[r\left(\frac{k_{1}}{\mu} \frac{\partial p}{\partial r}+\frac{k_{5}}{\mu} \frac{\partial p}{\partial z}\right)\right]+\frac{\partial}{\partial z}\left(\frac{k_{5}}{\mu} \frac{\partial p}{\partial r}+\frac{k_{3}}{\mu} \frac{\partial p}{\partial z}\right)\right\}=0
\end{gathered}
$$

where the coefficient $M$ is obtained from equation (4), $k_{4}=k_{6}=0$ for $r, z$-symmetric monoclinic materials and equations (9) under axisymmetric conditions are employed.

\subsection{Energy Conservation Equation}

The equation of energy conservation which governs the balance of energy in a control volume is written as 


$$
\frac{\partial E}{\partial t}+\nabla \cdot \mathbf{q}=\frac{\partial E^{g e n}}{\partial t}
$$

where on the left hand side, the first term is the storage term which represents the rate at which energy is stored within the control volume and the second term is the diffusion term which defines the flux of energy convecting through the control volume and the right-hand side represents the rate at which energy is being generated or consumed due to the process.

The rate of the energy stored in the control volume is the sum of the rate of the energy stored in the solid and gas. The first term of equation (22) may be expressed as

$$
\frac{\partial E}{\partial t}=\left[(1-\phi) \rho_{s}\left(C_{p}\right)_{s}+\phi \rho_{g}\left(C_{p}\right)_{g}\right]\left(\frac{\partial T}{\partial t}\right)
$$

where the subscripts $s$ and $g$ stand for solid and gas, respectively.

The energy diffusion term consists of two contributions: heat conduction in the bulk solid and heat convection carried by the gas flow through the pores.

$$
\mathbf{q}=\mathbf{q}_{\text {cond }}+\mathbf{q}_{\text {conv }}
$$

The heat conduction term can be written by the Fourier conduction law for anisotropic materials as

$$
\mathbf{q}_{\text {cond }}=-\kappa \cdot \nabla T
$$

where $\kappa$, the conductivity matrix, is similar in form to equation (16). The convective heat flux is the gas enthalpy $h_{\mathrm{g}}$ times the gas mass flux $\rho_{\mathrm{g}} \mathbf{v}_{\mathrm{g}}$ and is written as

$$
\mathbf{q}_{\text {conv }}=h_{g} \rho_{g} \mathbf{v}_{g}-\left(C_{p}\right){ }_{g} \rho_{g} \mathbf{v}_{g} T
$$

where the final term is valid if the gas is assumed to be ideal. Substituting equations (25) and (26) into (24) and taking the divergence of the result gives

$$
\nabla \cdot \mathbf{q}=-\nabla \cdot(\mathbf{k} \cdot \nabla T)-\rho_{g}\left(C_{p}\right)_{g} \nabla T \cdot\left(\frac{1}{\mu} \mathbf{k} \cdot \nabla p\right)-\rho_{g} h_{g} \nabla \cdot\left(\frac{1}{\mu} \mathbf{k} \cdot \nabla p\right)
$$

where the second and first forms of (26) are operated upon to get the second and third terms, respectively, and (15) is recalled. The reason for this juggling act is to strengthen 
coupling between variables yet, from intuition, circumvent nonlinearities where possible. Notably, the last term does not appear in Sullivan and Salamon [3] because gas velocity was assumed to be locally constant. However for the problems involving thermal gradients, see [12], both of these terms have a significant effect on the process.

The energy density due to the heat generated in the control volume is expressed by the product of the virgin material density $\rho_{\text {virg }}$, the heat of reaction $h_{R}$, and the rate of the process

$d c_{1} / d t$ as

$$
\frac{\partial E^{g e n}}{\partial t}=-\rho_{v i r g} h_{R} \frac{d c_{1}}{d t}
$$

Substitution of equations (23), (27) and (28) into equation (22) yields

$$
\begin{gathered}
{\left[(1-\phi) \rho_{s}\left(C_{p}\right)_{s}+\phi \rho_{g}\left(C_{p}\right)_{g}\right] \frac{\partial T}{\partial t}-\nabla \cdot(\kappa \cdot \nabla T)} \\
-\rho_{g}\left(C_{p}\right)_{g} \nabla T \cdot\left(\frac{1}{\mu} \mathbf{k} \cdot \nabla p\right)-\rho_{g} h_{g} \nabla \cdot\left(\frac{1}{\mu} \mathbf{k} \cdot \nabla p\right)+\rho_{v i r g} h_{R} \frac{d c_{1}}{d t}=0
\end{gathered}
$$

In the energy conservation equation (29), both pressure and temperature are independent variables. The nonlinear term ( $3 \mathrm{rd}$ term) can be linearized by choosing either pressure or temperature as a variable parameter and this choice is discussed below equation (32).

Axisymmetric case. The energy conservation equation (29) for the axisymmetric case in cylindrical coordinates is written as

$$
\begin{gathered}
\frac{\partial}{\partial t}\left(\left[(1-\phi) \rho_{s}\left(C_{p}\right)_{s}+\phi \rho_{g}\left(C_{p}\right)_{g}\right] T_{g}\right)+\rho_{v i r g} h_{R} \frac{d c_{1}}{d t} \\
-\frac{1}{r} \frac{\partial}{\partial r}\left[r\left(\kappa_{1} \frac{\partial T}{\partial r}+\kappa_{5} \frac{\partial T}{\partial z}\right)\right]-\frac{\partial}{\partial z}\left(\kappa_{5} \frac{\partial T}{\partial r}+\kappa_{3} \frac{\partial T}{\partial z}\right) \\
-\rho_{g}\left(C_{p}\right)_{g}\left[\left(\frac{k_{1}}{\mu} \frac{\partial p}{\partial r}+\frac{k_{5}}{\mu} \frac{\partial p}{\partial z}\right) \frac{\partial T}{\partial r}+\left(\frac{k_{5}}{\mu} \frac{\partial p}{\partial r}+\frac{k_{3}}{\mu} \frac{\partial p}{\partial z}\right) \frac{\partial T}{\partial z}\right] \\
-\rho_{g} h_{g}\left\{\frac{1}{r} \frac{\partial}{\partial r}\left[r\left(\frac{k_{1}}{\mu} \frac{\partial p}{\partial r}+\frac{k_{5}}{\mu} \frac{\partial p}{\partial z}\right)\right]+\frac{\partial}{\partial z}\left(\frac{k_{5}}{\mu} \frac{\partial p}{\partial r}+\frac{k_{3}}{\mu} \frac{\partial p}{\partial z}\right)\right\}=0
\end{gathered}
$$


Because this equation employs the final term in equation (26), it is restricted by the assumption of an ideal gas.

\section{AXISYMMETRIC FINITE ELEMENT FORMULATION}

The axisymmetric finite element equations are obtained by successively applying the Galerkin method to equations (11), (21) and (30) in the following form:

$$
\left.\int_{A} N_{i} \text { (LHS_of_referenced_equation }\right) r d A=0
$$

where LHS denotes the left hand side and $\mathrm{A}$ is the domain in the $\mathrm{r}, \mathrm{z}$ plane.

The nonlinear, penultimate term in equation (30), namely,

$$
-\rho_{g}\left(C_{p}\right)_{g}\left[\left(\frac{k_{1}}{\mu} \frac{\partial p}{\partial r}+\frac{k_{5}}{\mu} \frac{\partial p}{\partial z}\right) \frac{\partial T}{\partial r}+\left(\frac{k_{5}}{\mu} \frac{\partial p}{\partial r}+\frac{k_{3}}{\mu} \frac{\partial p}{\partial z}\right) \frac{\partial T}{\partial z}\right]
$$

is linearized by choosing the spatial derivatives of temperature as parameters since pressure is a more sensitive factor to the processes studied. Then applying Green's theorem, and integrating (31) and (32) by parts (Lee, 1993), the following matrix equation is obtained.

$$
[C] \frac{d}{d t}\{a\}+[K]\{a\}=\{F\}
$$

where

$$
\begin{array}{cc}
{[C]=\left[\begin{array}{cccc}
0 & 0 & 0 & 0 \\
0 & 0 & 0 & 0 \\
C_{p u} C_{p w} C_{p p} & C_{p T} \\
0 & 0 & 0 & C_{T T}
\end{array}\right] \quad[K]=\left[\begin{array}{cccc}
K_{u u} & K_{u w} & K_{u p} & K_{u T} \\
K_{w u} & K_{w w} & K_{w p} & K_{w T} \\
0 & 0 & K_{p p} & 0 \\
0 & 0 & K_{T p} & K_{T T}
\end{array}\right]} \\
& \{F\}=\left[F_{u} F_{w} F_{p} F_{T}\right] \quad\{a\}=[u w p T]
\end{array}
$$

The elements of $[C],[K]$ and $\{F\}$ are explicitly written in the Appendix. 
It should be noted that the coupling term $K_{\text {Tp }}$ does not appear in Sullivan and Salamon [3]. They treat the spatial derivatives of pressure as parameters which causes these terms to be deposited on the diagonal of the $[\mathrm{K}]$ matrix rather than in the $\mathrm{K}_{\mathrm{Tp}}$ term. The above formulation results in a more strongly coupled system of equations and is employed here.

For integration over time, the variably weighted Euler numerical method [14] is employed which leads to

$$
\left[K_{e f f}\right]^{n}\{a\}^{n+1}=\left\{F_{e f f}\right\}^{n}
$$

where

$$
\begin{gathered}
{\left[K_{e f f}\right]^{n}=\frac{1}{\Delta t^{n}}[C]^{n}+\theta[K]^{n}-\theta[H]^{n}} \\
\left\{F_{e f f}\right\}^{n}=\{F\}^{n}+\left[\frac{1}{\Delta t^{n}}[C]^{n}-(1-\theta)[K]^{n}-\theta[H]^{n}\right]\{a\}^{n}
\end{gathered}
$$

and superscript $n$ denotes the time step. In equations (36) and (37), the matrix $[H]$ may be defined as

$$
[H]^{n}=\left(\frac{\partial\{F\}}{\partial\{a\}}\right)^{n}
$$

For solution by the fully implicit time integration scheme, the matrix $[H]$ is required. Details of its formulation are given in the dissertation by Lee [12]. Nonzero elements of $[H]$ are given in the Appendix.

In equation (35), $\left[K_{\text {eff }}\right]^{\mathrm{n}}$ and $\left\{F_{\text {eff }}\right\}^{\mathrm{n}}$ are the effective stiffness matrix and effective force vector at time step $t_{\mathrm{n}}$ respectively. To obtain the material displacements, pore pressure and temperature at each time step, equation (35) is solved for $\{a\}^{\mathrm{n}+1}$ simultaneously using a factorization procedure which is a version of Gauss elimination with partial pivoting [15]. 


\section{DECOMPOSING CARBON PHENOLICS}

The governing equations for poroelastic material with thermal and gas diffusion provided in Section 2 are applicable to thermochemically decomposing carbon-phenolic. The momentum equations remain the same, but the generation terms in the gas diffusion and the energy conservation equations require further specialization. The objective is to specialize and apply the linearized theory to models of carbon-phenolic material and simulate laboratory tests in order to demonstrate it and establish poroelastic parameters over a nonlinear decomposition process.

\subsection{Governing Equations}

For the mass generation term (19) in the gas diffusion equation (12), the rate of gas mass accumulation is due to decomposition reactions and, from Sullivan and Salamon [3], is given by

$$
\frac{\partial m_{g}^{g e n}}{\partial t}=-\sum_{i=1}^{N} R F \rho_{v i r g}\left[\left(W_{O}\right)_{i}-\left(W_{C}\right)_{i}\right] \frac{d\left(c_{1}\right)_{i}}{d t}
$$

where $R F$ is the weight fraction of the virgin composite which is resin, $\rho_{\text {virg }}$ is the virgin density of the composite, $\left(W_{O}\right)_{i}$ is the fractional weight of the resin which undergoes the $i^{\text {th }}$ reaction, $\left(W_{\mathrm{C}}\right)_{\mathrm{i}}$ is the fractional weight of the resin which is left as a solid residue by the $i^{\text {th }}$ reaction, and $N$ is the number of reactions. Following [3], the rate of charring $d\left(c_{1}\right)_{\mathrm{i}} / d t$ is expressed by the Arrhenius kinetic reaction equation

$$
\frac{d\left(c_{1}\right)_{i}}{d t}=-\left(A^{s}\right)_{i}\left(c_{1}\right)_{i}^{\left(n^{s}\right)_{i}} \exp \left[-\frac{\left(E_{a}^{s}\right)_{i}}{R T}\right]
$$

where $A^{\mathrm{S}}$ and $n^{\mathrm{S}}$ are the Arrhenius constants and $E_{\mathrm{a}}^{\mathrm{S}}$ is the activation energy for the chemical reaction. These constants for carbon-phenolic are listed in Table 1. 
In this work, pyrolysis gases are treated as ideal and the gas density $\rho_{\mathrm{g}}$ in the governing equations is obtained by the ideal gas law

$$
\rho_{g}=\frac{M W_{g}}{R T} p
$$

where $M W_{\mathrm{g}}$ is the molecular weight of gas and $R$ is the universal gas constant. The mechanical and thermal properties of dry carbon-phenolic are listed in Table 2.

\subsection{RTG and FTE Tests and Their Finite Element Models}

Stokes [10] conducted two high temperature experiments on cylindrical carbonphenolic specimens (dry FM5055), fabricated so that the plane of the carbon fabric is perpendicular to the longitudinal axis of the specimen, in order to determine thermomechanical behavior during chemical decomposition. The restrained thermal growth (RTG) test measured both the stress required to hold the specimens at a constant longitudinal strain and the resulting lateral strain. The free thermal expansion (FTE) test measured the resulting longitudinal strain. The specimens were heated uniformly at a rate of 5.55 degrees Kelvin per second (10 degrees Fahrenheit per second), and this was controlled using thermocouples embedded in the specimens.

The geometry, coordinate system and finite element mesh for the RTG and FTE test specimens are shown in Figure 1. In the RTG test, it was assumed that the end constraints prevent pyrolysis gases from flowing along the axial $z$-direction. Hence, impermeable conditions $\partial p / \partial z=0$ are prescribed at $z=0$ and $z=b$. However in the FTE test, the pyrolysis gases do flow along the $z$-direction, hence to allow the gases to escape, atmospheric pressure is specified at $z=0$ and $z=b$. The initial conditions are $T=293$ degrees Kelvin and $p=1 \mathrm{~atm}$ in the model and the boundary conditions are summarized in Table 3 .

The energy generation term (28) in equation (22) is simplified in order to maintain a uniform temperature increase in the model. An internal heat source is prescribed in each finite element through the integral equation 


$$
\frac{\partial E^{g e n}}{\partial t} \leftarrow\left(Q_{i n t}\right)_{i}=\int_{A} N_{i} q_{i n t} r d A
$$

where $q_{\text {int }}$ is the heat generation per unit area, and $\left(Q_{\text {int }}\right)_{\mathrm{i}}$ is an equivalent nodal value. The value of $q_{\text {int }}$, found by trial and error in order to achieve the rate of temperature increase of the specimen ( 5.55 degrees Kelvin per second), was determined to be $11.4 \times 10^{6} \mathrm{Joulel}$ $m^{3} \sec$.

\subsection{Results for the pressure-stress coupling factors $\alpha$}

Calculation of the pressure-stress coupling factors from equations (5) and (6) for the virgin elastic composite material requires an experiment, the Biot and Willis $[5,6]$ unjacketed test. Unfortunately such an experiment for carbon-phenolic has not been conducted. Moreover after decomposition commences, (5) and (6) are suspect because of severe changes in material morphology and properties. Hence further experiments would be desireable. In short, requisite data to calculate these poroelastic parameters does not exist.

Results are obtained by simulating the RTG and FTE tests and varying $\alpha$, then comparing the material response to experimental data. This was done by physical intuition and trial and error based upon a hypothesis introduced by Lee [12]. This hypothesis assumes (1) isotropic porosity, i.e., $\alpha_{i}=\alpha, i=1,2,3,5$, (2) a higher proportion of closed pores in virgin material, and (3) increased pore channel opening under high shearing stress action. Consequently, the pressure-stress coupling factor (1) is initially high and, if data is available, can be determined from material properties and equations (5) and (6), (2) decreases as porosity opens, thus inversely as permeability increases, and (3) is further decreased if shear stress enhances pore channel growth. (Note: the procedure employed here is to guess $\alpha$, then use equations (6) and (4) to compute $M$; hence $\alpha$ is a curve fit parameter.)

In Figures 2 and 3, average restraining stress and average lateral strain of the RTG test simulation are plotted versus average temperature. Also, in Figure 4, average longitu- 
dinal strain of the FTE test simulation is plotted versus average temperature. The variation with temperature of the pressure-stress coupling factor $\alpha$ used in the RTG and FTE simulations, shown in Figure 5, is that for which the numerical solutions most closely match the measured responses. Only values at corners of the curves were adjusted, those in between corner values were linearly interpolated. The pressure-stress coupling factor for the RTG test simulation was decreased more than that for the FTE test simulation due to a higher shear stress level in the RTG test simulation. Notably for the RTG test simulation, a single choice for the varying pressure-stress coupling factor correlates with data for both restraining stress and lateral strain; Sullivan and Salamon [3] required different constant values to achieve satisfactory results in their parametric study.

\section{CONCLUSION}

A coupled set of governing equations for a poroelastic solid with thermal and mass diffusion is derived in a practicable form which requires a minimum of experimental data, yet are sufficiently realistic to entertain engineering problems. In the energy conservation equation, both conductive heat transfer in the bulk solid and convective heat transfer carried by the diffusive gas through the bulk porous solid are treated. The convection terms in which pressure is the independent variable are included in the energy conservation equation so that the convection heat transfer by the diffusive gas is accounted for more accurately than in [3]. These convection terms are coupled with the gas mass diffusion term in the gas diffusion equation, and therefore pressure appears in both the gas diffusion equation and the energy conservation equation as an independent variable. Hence the theory now handles the thermal and gas diffusion in the poroelastic solid in a strongly coupled manner.

The material formulation includes the most general anisotropic material under the constraints of axisymmetry, and therefore permits application to realistic, three-dimensional composite structures. Importantly the axisymmetric formulation permits accurate computation of the transverse shear stress which is hypothesized to play a significant role in pore channel opening during material decomposition and in turn enables finer definition 
of the pressure-stress coupling factor during decomposition.

The coupled theory, when applied to carbon-phenolic material tests, provides consistent and close correlations with the experimental data using an empirically determined pressure-stress coupling factor suggested by Lee [12]. The improved correlation with experimental data in simulating these tests is attributed to the new formulation for the pressure-stress coupling factor.

It is anticipated that the strong coupling of the equations will play a major role in accurate computation of spatially nonuniform thermal problems such as those encountered in rocket liner structures during firing.

\section{ACKNOWLEDGMENTS}

The first and the second authors are grateful for support through NASA Research Grant NAG8-879. The third author acknowledges support from the Marshall Space Flight Center.

\section{REFERENCES}

${ }^{1}$ Bazant, Z., Mechanics of Geomaterials, John Wiley and Sons Ltd., 1985.

${ }^{2}$ Sullivan, R.M., "A Finite Element Model for Thermochemically Decomposing Polymers," Ph.D. Dissertation, The Pennsylvania State University, 1990.

${ }^{3}$ Sullivan, R.M. and Salamon, N.J., "A Finite Element Method for the Thermochemical Decomposition of Polymeric Materials-Part I: Theory," International Journal of Engineering Science, Vol. 30, No. 4, 1992, pp. 431-441.

${ }^{4}$ Weiler, F.C., "Fully Coupled Thermo- Poro- Elasto Governing Equations," in Computational Mechanics of Porous Materials and Their Thermal Decomposition, N.J. Salamon and R.M. Sullivan, Edts., AMD-Vol. 136, ASME Press, 1992.

${ }^{5}$ Biot, M.A., "General Theory of Three-Dimensional Consolidation," Journal of Applied Physics, Vol. 12., February, 1941, pp. 155-164. 
${ }^{6}$ Biot, M.A. and Willis, D.G., "The Elastic Coefficients of the Theory of Consolidation," ASME Journal of Applied Mechanics, Vol. 24, December, 1957, pp. 596-601.

${ }^{7}$ Nur, A. and Byerlee, J.D., "An Exact Effective Stress Law for Elastic Deformation of Rock with Fluids," Journal of Geophysical Research, Vol. 76, No. 26, 1971, pp. 6414-6419.

${ }^{8}$ Carroll, M.M., "An Effective Stress Law for Anisotropic Elastic Deformations," Journal of Geophysical Research, Vol. 84, No. B13, 1979, 7510-7512.

${ }^{9}$ Kurashige, M., "A Thermoelastic Theory of Fluid-Filled Porous Materials," International Journal of Solids and Structures, Vol. 25, No. 9, 1989, pp. 1039-1052.

${ }^{10}$ Stokes, E.H., "The Effect of Moisture on the Mechanical and Thermal Response of FM5055 Carbon Phenolic Composites," Southern Research Institute Report No. SRIEAS-87-1244-6245-6, Southern Research Institute, Birmingham, AL. 1987.

${ }^{11}$ Rudnicki, J.W., "Effect of Pore Fluid Diffusion on Deformation and Failure of Rock," pp. 315-347, in Mechanics of Geomaterials, Z. Bazant, Edt., John Wiley and Sons Ltd., 1985.

${ }^{12}$ Lee, S., "Coupled Finite Element Analysis of Decomposing Polymeric Composites and Structures with Subsequent Thermal and Gas Diffusion," Ph.D. Dissertation, The Pennsylvania State University, 1993.

${ }^{13}$ Kansa, E.J., Perlee, H.E. and Chaiken, R.F., "Mathematical Model of Wood Pyrolysis Including Internal Forced Convection," Combustion and Flame, Vol. 29, September, 1977, pp. 311-324.

${ }^{14}$ Celia, M.A. and Gray, W.G., Numerical Methods for Differential Equations, Prentice Hall, Englewood Cliffs, 1992.

${ }^{15}$ Dongarra, J.J., Moler, C.B., Bunch, J.R. and Stewart, G.W., LINPACK Users' Guide, SIAM, 1979. 


\section{APPENDLX}

The elements of $[C],[K]$ and $\{F\}$ are

$$
\begin{aligned}
& \left(K_{u u}\right)_{i j}=\int_{A} N_{i, r}\left(C_{11} N_{j, r}+C_{12} \frac{1}{r} N_{j}+C_{15} N_{j, z}\right) r d A \\
& +\int_{A} N_{i, z}\left(C_{15} N_{j, r}+C_{25} \frac{1}{r} N_{j}+C_{55} N_{j, z}\right) r d A \\
& +\int_{A} N_{i}\left(C_{12} N_{j, r}+C_{22} \frac{1}{r} N_{j}+C_{25} N_{j, z}\right) d A \\
& \left(K_{u w}\right)_{i j}=\int_{A} N_{i, r}\left(C_{15} N_{j, r}+C_{13} N_{j, z}\right) r d A+\int_{A} N_{i, z}\left(C_{55} N_{j, r}+C_{35} N_{j, z}\right) r d A \\
& +\int_{A} N_{i}\left(C_{25} N_{j, r}+C_{23} N_{j, z}\right) d A \\
& \left(K_{u p}\right)_{i j}=-\int_{A} N_{i, r} \alpha_{1} N_{j} r d A-\int_{A} N_{i, z} \alpha_{5} N_{j} r d A-\int_{A} N_{i} \alpha_{2} N_{j} d A \\
& \left(K_{u T}\right)_{i j}=-\int_{A} N_{i, r} Q_{1} N_{j} r d A-\int_{A} N_{i, z} Q_{5} N_{j} r d A-\int_{A} N_{i} Q_{2} N_{j} d A \\
& \left(K_{w u}\right)_{i j}=\int_{A} N_{i, r}\left(C_{15} N_{j, r}+C_{25} \frac{1}{r} N_{j}+C_{55} N_{j, z}\right) r d A \\
& +\int_{A} N_{i, r}\left(C_{13} N_{j, r}+C_{23} \frac{1}{r} N_{j}+C_{35} N_{j, z}\right) r d A \\
& \left(K_{w w}\right)_{i j}=\int_{A} N_{i, r}\left(C_{55} N_{j, r}+C_{35} N_{j, z}\right) r d A+\int_{A} N_{i, z}\left(C_{35} N_{j, r}+C_{33} N_{j, z}\right) r d A \\
& \left(K_{w p}\right)_{i j}=-\int_{A} N_{i, r} \alpha_{5} N_{j} r d A-\int_{A} N_{i, z} \alpha_{3} N_{j} r d A \\
& \left(K_{w T}\right)_{i j}=-\int_{A} N_{i, r} Q_{5} N_{j} r d A-\int_{A} N_{i, 2} Q_{3} N_{j} r d A \\
& \left(K_{p p}\right)_{i j}=\int_{A} N_{i, r} \rho_{g}\left(\frac{k_{1}}{\mu} N_{j, r}+\frac{k_{5}}{\mu} N_{j, z}\right) r d A+\int_{A} N_{i, z} \rho_{g}\left(\frac{k_{5}}{\mu} N_{j, r}+\frac{k_{3}}{\mu} N_{j, z}\right) r d A
\end{aligned}
$$




$$
\begin{aligned}
& \left(K_{T p}\right)_{i j}=-\rho_{g}\left(C_{p}\right)_{g} \frac{\partial T}{\partial r} \int_{A} N_{i}\left(\frac{k_{1}}{\mu} N_{j, r}+\frac{k_{5}}{\mu} N_{j, z}\right) r d A \\
& -\rho_{g}\left(C_{p}\right) \frac{\partial T}{g} \int_{A} N_{i}\left(\frac{k_{5}}{\mu} N_{j, r}+\frac{k_{3}}{\mu} N_{j, z}\right) r d A+\rho_{g} h_{g} \int_{A} N_{i, r}\left(\frac{k_{1}}{\mu} N_{j, r}+\frac{k_{5}}{\mu} N_{j, z}\right) r d A \\
& +\rho_{g} h_{g} \int_{A} N_{i, z}\left(\frac{k_{5}}{\mu} N_{j, r}+\frac{k_{3}}{\mu} N_{j, z}\right) r d A \\
& \left(K_{T T}\right)_{i j}=\int_{A} N_{i, r}\left(\kappa_{1} N_{j, r}+\kappa_{5} N_{j, z}\right) r d A+\int_{A} N_{i, z}\left(\kappa_{5} N_{j, r}+\kappa_{3} N_{j, z}\right) r d A \\
& \left(C_{p u}\right)_{i j}=\int_{A} N_{i} \rho_{g}\left(\alpha_{1} N_{j, r}+\alpha_{5} N_{j, z}\right) r d A+\int_{A} N_{i} \alpha_{2} \rho_{g} N_{j} d A \\
& \left(C_{p w}\right)_{i j}=\int_{A} N_{i} \rho_{g}\left(\alpha_{5} N_{j, r}+\alpha_{3} N_{j, z}\right) r d A \\
& \left(C_{p p}\right)_{i j}=\int_{A} N_{i} \frac{\rho_{g}}{M} N_{j} r d A \\
& \left(C_{p T}\right)_{i j}=-\int_{A} N_{i} \rho_{g}\left(\alpha_{1} \beta_{1}+\alpha_{2} \beta_{2}+\alpha_{3} \beta_{3}+\alpha_{5} \beta_{5}+\phi \beta_{g}\right) N_{j} r d A \\
& \left(C_{T T}\right)_{i j}=\int_{A} N_{i}\left[(1-\phi) \rho_{s}\left(C_{p}\right)_{s}+\phi \rho_{g}\left(C_{p}\right)_{g}\right] N_{j} r d A \\
& \left(F_{u}\right)_{i}=\int_{s_{u}} N_{i} t_{r}^{*} r d s \\
& \left(F_{w}\right)_{i}=\int_{s_{w}} N_{i} t_{w}^{*} r d s \\
& \left(F_{p}\right)_{i}=-\left(\rho_{v i r g}-\rho_{p r o c}\right) \int_{A} N_{i}\left(\dot{c}_{1}\right) r d A-\rho_{g} \int_{s_{p}} N_{i}\left(v_{g}^{*}\right)_{k} n_{k} r d s \\
& \left(F_{T}\right)_{i}=-\rho_{v i r g} h_{R} \int_{A} N_{i} \dot{c}_{1} r d A-\int_{s_{T}} N_{i}\left(q^{*}\right)_{k} n_{k} r d s
\end{aligned}
$$

where

$$
\begin{array}{ll}
Q_{1}=C_{11} \beta_{1}+C_{12} \beta_{2}+C_{13} \beta_{3}+C_{15} \beta_{5} & Q_{2}=C_{12} \beta_{1}+C_{22} \beta_{2}+C_{23} \beta_{3}+C_{25} \beta_{5} \\
Q_{3}=C_{13} \beta_{1}+C_{23} \beta_{2}+C_{33} \beta_{3}+C_{35} \beta_{5} & Q_{5}=C_{15} \beta_{1}+C_{25} \beta_{2}+C_{35} \beta_{3}+C_{55} \beta_{5}
\end{array}
$$

and where $v_{\mathrm{g}}{ }^{*}$ is the volume average gas velocity specified along the external boundary of 
arc length $s_{\mathrm{p}}, q^{*}$ is the heat flux specified on $s_{\mathrm{T}}, n_{\mathrm{i}}$ represents components of the outward unit vector normal to the boundaries, and $t_{\mathrm{r}}{ }^{*}$ and $t_{\mathrm{z}}{ }^{*}$ represent traction components distributed over the external boundary of arc length $s_{\mathrm{u}}$ and $s_{\mathrm{w}}$ in the $r$ and $z$ directions, respectively.

The $[\mathrm{H}]$ matrix is written explicitly as

$$
[H]=\left[\begin{array}{llll}
0 & 0 & 0 & 0 \\
0 & 0 & 0 & 0 \\
0 & 0 & 0 & H_{p T} \\
0 & 0 & 0 & H_{T T}
\end{array}\right]
$$

where

$$
\begin{gathered}
\left(H_{p T}\right)_{i j}=-\left(\rho_{v i r g}-\rho_{c h a r}\right) \int_{A}\left(\frac{\partial \dot{c}_{1}}{\partial T}\right) N_{i} N_{j} r d A \\
\left(H_{p T}\right)_{i j}=-\rho_{v i r g} h_{R} \int_{A}\left(\frac{\partial \dot{c}_{1}}{\partial T}\right) N_{i} N_{j} r d A
\end{gathered}
$$


Table 1: Constants in the decomposition model for dry carbon-phenolic.

\begin{tabular}{|c|l|r|r|r|r|}
\hline $\begin{array}{c}\text { Reaction } \\
\text { number (i) }\end{array}$ & $E_{a}^{s}(J /$ mole $)$ & $A^{s}(1 / \mathrm{sec})$ & $n^{s}$ & \multicolumn{1}{|c|}{$W_{O}$} & $W_{C}$ \\
\hline \hline 1 & 88764.4 & $1.207305 \times 10^{10}$ & 3.5 & 0.0015 & 0 \\
\hline 2 & 117236 & $4.057500 \times 10^{9}$ & 6.5 & 0.095 & 0 \\
\hline 3 & 211443.5 & $3.857777 \times 10^{14}$ & 6.5 & 0.59 & 0.29 \\
\hline 4 & 272155 & $5.583611 \times 10^{15}$ & 3.3 & 0.3 & 0.19 \\
\hline
\end{tabular}

$\mathrm{RF}=0.3, h_{\mathrm{R}}=0, M W_{\mathrm{g}}=0.03 \mathrm{~kg} / \mathrm{mole}$ 
Table 2 : Mechanical and thermal properties of dry carbon-phenolic.

\begin{tabular}{|c|c|c|c|}
\hline \multirow{2}{*}{ property } & \multicolumn{3}{|c|}{ temperature range } \\
\cline { 2 - 4 } & $\mathrm{T}<450^{\circ} \mathrm{K}$ & $450^{\circ} \mathrm{K}<\mathrm{T}<533^{\circ} \mathrm{K}$ & $\mathrm{T}>533^{\circ} \mathrm{K}$ \\
\hline $\mathrm{E}_{\mathrm{L}}$ & $1.517 \times 10^{10} \mathrm{~Pa}$ & $-1.412 \times 10^{8} \mathrm{~T}+7.87 \times 10^{10} \mathrm{~Pa}$ & $3.447 \times 10^{9} \mathrm{~Pa}$ \\
\hline $\mathrm{E}_{\mathrm{T}}$ & $1.793 \times 10^{10} \mathrm{~Pa}$ & $\begin{array}{c}-1.7449 \times 10^{8} \mathrm{~T}+9.645 \times 10^{10} \\
\mathrm{~Pa}\end{array}$ & $3.447 \times 10^{9} \mathrm{~Pa}$ \\
\hline$v_{\mathrm{TL}}$ & 0 & $-0.00343 \mathrm{~T}+1.83819$ & 0 \\
\hline$v_{\mathrm{LT}}$ & 0 & $-0.00289 \mathrm{~T}+1.551$ & 0 \\
\hline$v_{\mathrm{T}}$ & 0 & 0 & 0 \\
\hline
\end{tabular}

\begin{tabular}{|c|c|c|c|}
\hline property & value & property & value \\
\hline$\beta_{\mathrm{T}}$ & $0.000006 \mathrm{~m} / \mathrm{m}^{-}{ }^{\circ} \mathrm{K}$ & $\mathrm{b}_{\mathrm{L}}$ & $0.000012 \mathrm{~m} / \mathrm{m}-{ }^{\circ} \mathrm{K}$ \\
\hline$\left(k_{\mathrm{T}}\right)_{\text {virg }} / \mu$ & $5 \times 10^{-21} \mathrm{~m}^{3}-\mathrm{sec} / \mathrm{kg}$ & $\left(k_{\mathrm{L}}\right)_{\text {virg }} / \mu$ & $5 \times 10^{-21} \mathrm{~m}^{3}-\mathrm{sec} / \mathrm{kg}$ \\
\hline$\left(k_{\mathrm{T}}\right)_{\text {char }} / \mu$ & $5 \times 10^{-13} \mathrm{~m}^{3}-\mathrm{sec} / \mathrm{kg}$ & $\left(k_{\mathrm{L}}\right)_{\text {char }} / \mu$ & $5 \times 10^{-13} \mathrm{~m}^{3}-\mathrm{sec} / \mathrm{kg}$ \\
\hline$\phi_{\text {virg }}$ & 0.02 & $\phi_{\text {char }}$ & 0.20 \\
\hline$\rho_{\text {virg }}$ & $1500.0 \mathrm{~kg} / \mathrm{m}$ & $\rho_{\text {char }}$ & $1300.0 \mathrm{~kg} / \mathrm{m}^{3}$ \\
\hline$\left(C_{\mathrm{p}}\right)_{\text {solid }}$ & $1400.0 \mathrm{~J} / \mathrm{kg}-{ }^{-} \mathrm{K}$ & $\left(C_{\mathrm{p}}\right)_{\text {gas }}$ & $1088.0 \mathrm{~J} / \mathrm{kg}-{ }^{\circ} \mathrm{K}$ \\
\hline$\kappa_{\text {solid }}$ & $1.44 \mathrm{~J} / \mathrm{m}-\mathrm{sec}-{ }^{\circ} \mathrm{K}$ & $\kappa_{\text {gas }}$ & $0.0 \mathrm{~J} / \mathrm{m}-\mathrm{sec}-{ }^{\circ} \mathrm{K}$ \\
\hline
\end{tabular}


Table 3 : Boundary conditions for the RTG and FTE test simulations.

\begin{tabular}{|c|c|c|}
\hline location & RTG Test & FTE Test \\
\hline$r=0$ & $u=\frac{\partial T}{\partial r}=\frac{\partial p}{\partial r}=0$ & $u=\frac{\partial T}{\partial r}=\frac{\partial p}{\partial r}=0$ \\
\hline$r=a$ & $\frac{\partial T}{\partial r}=0, p=1 \mathrm{~atm}$ & $\frac{\partial T}{\partial r}=0, p=1 \mathrm{~atm}$ \\
\hline$z=0$ & $w=\frac{\partial T}{\partial z}=\frac{\partial p}{\partial z}=0$ & $w=\frac{\partial T}{\partial z}=0, p=1 \mathrm{~atm}$ \\
\hline$z=b$ & $w=\frac{\partial T}{\partial z}=\frac{\partial p}{\partial z}=0$ & $\frac{\partial T}{\partial z}=0, p=1 \mathrm{~atm}$ \\
\hline
\end{tabular}




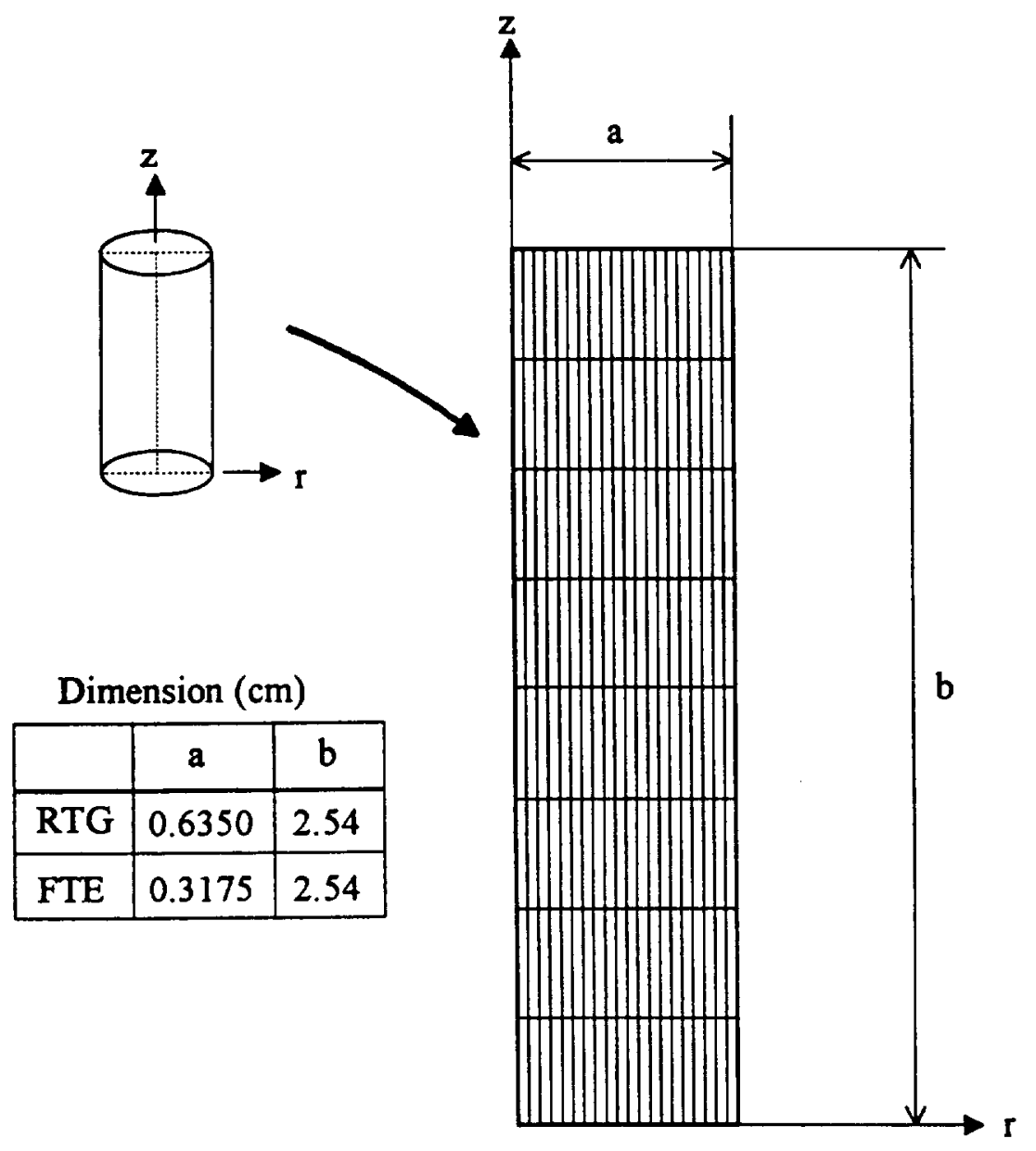

Figure 1 Finite element mesh for the RTG and FTE test simulations. 


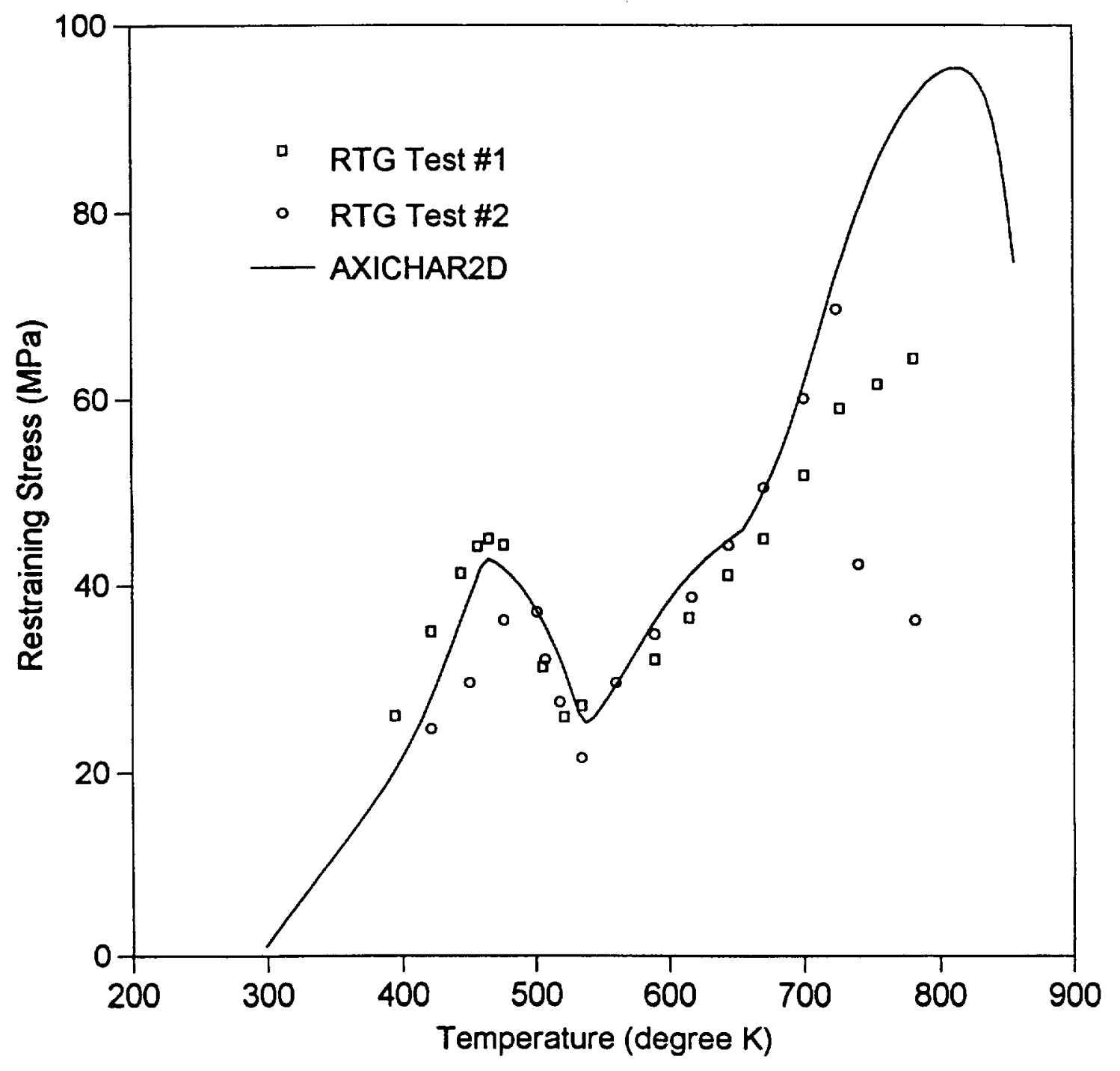

Figure 2 Restraining stress versus temperature in the RTG test simulation for varying pressure-stress coupling factor. 


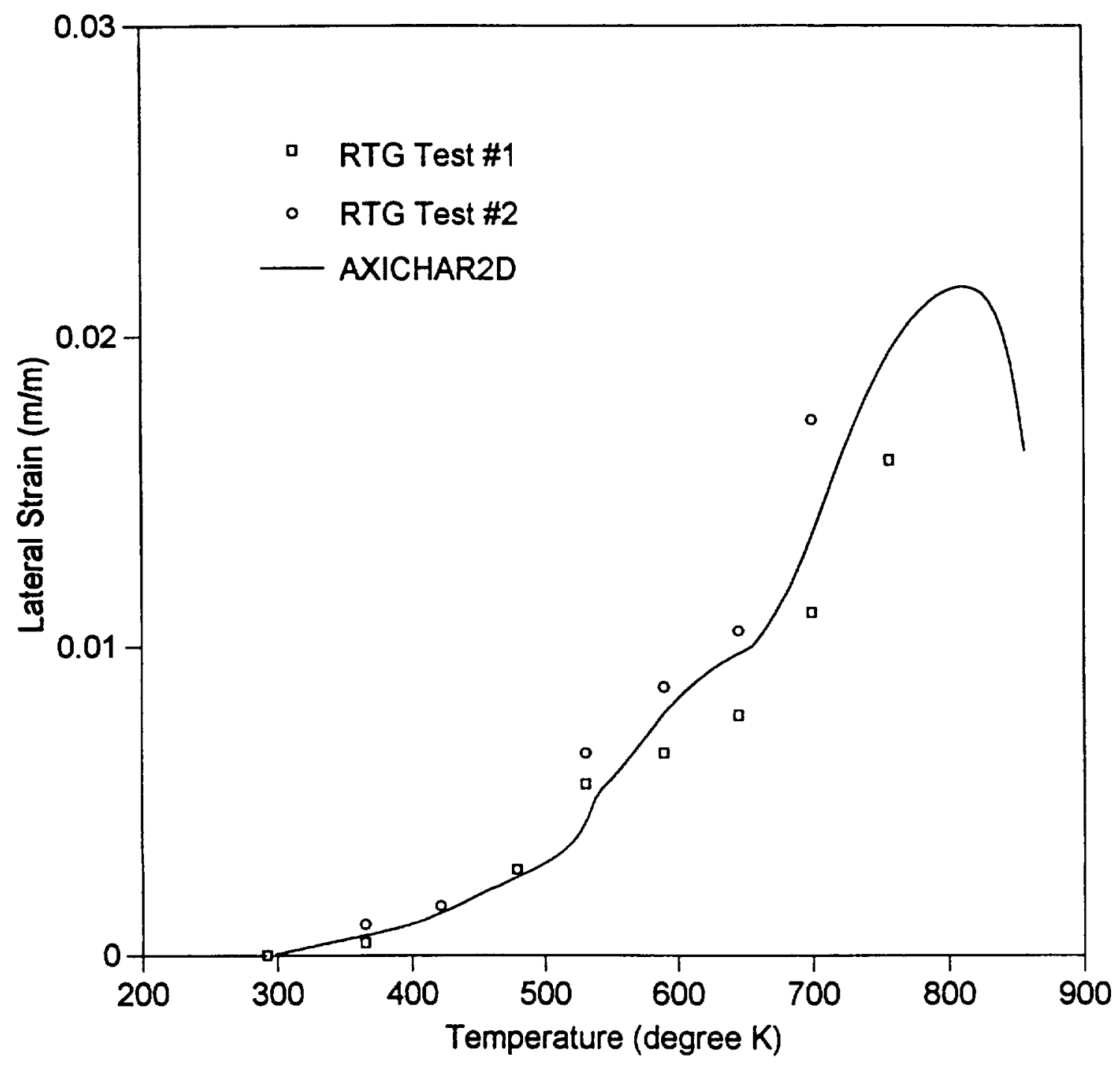

Figure 3 Lateral strain versus temperature in the RTG test simulation for varying pressure-stress coupling factor. 


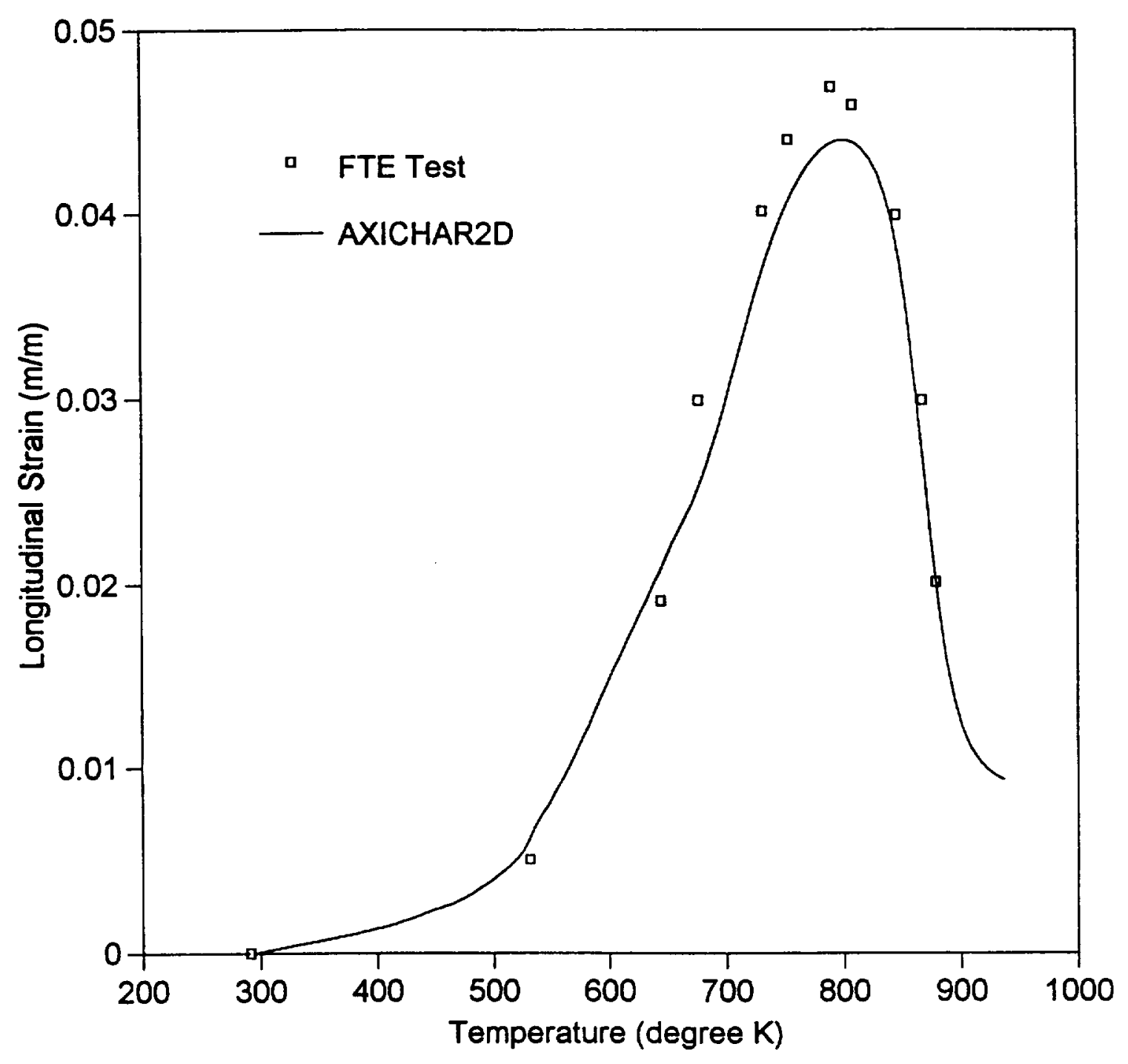

Figure 4 Longitudinal strain versus temperature in the FTE test simulation for varying pressure-stress coupling factor. 


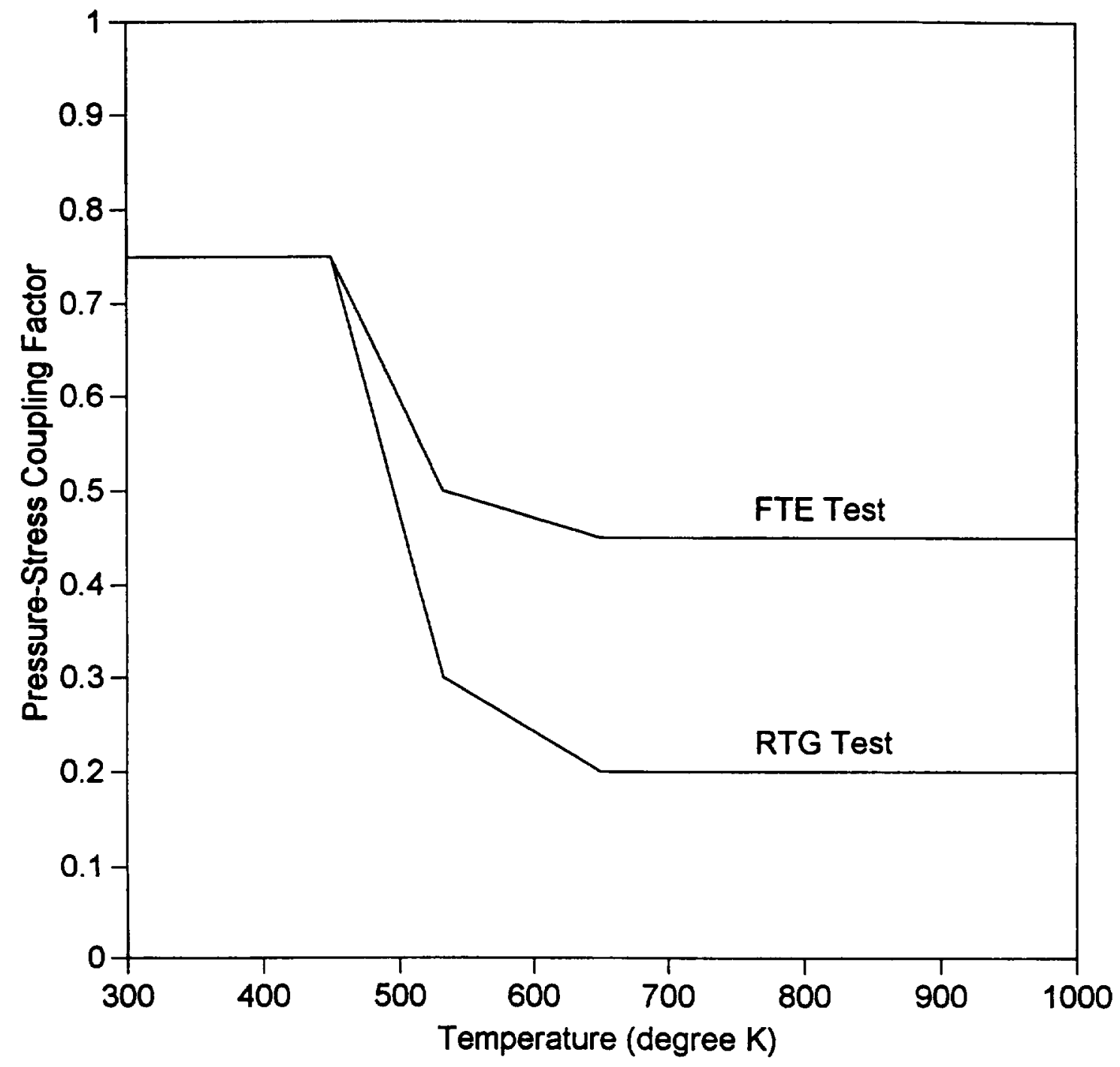

Figure 5 Typical variation of the pressure-stress coupling factor with temperature for the RTG and FTE test simulations. 


\section{LIST OF FIGURES}

Figure 1: Finite element mesh for the RTG and FTE test simulations.

Figure 2: Restraining stress versus temperature in the RTG test simulation for varying pressure-stress coupling factor.

Figure 3: Lateral strain versus temperature in the RTG test simulation for varying pressure-stress coupling factor.

Figure 4: Longitudinal strain versus temperature in the FTE test simulation for varying pressure-stress coupling factor.

Figure 5: Typical variation of the pressure-stress coupling factor with temperature for the RTG and FTE test simulations. 


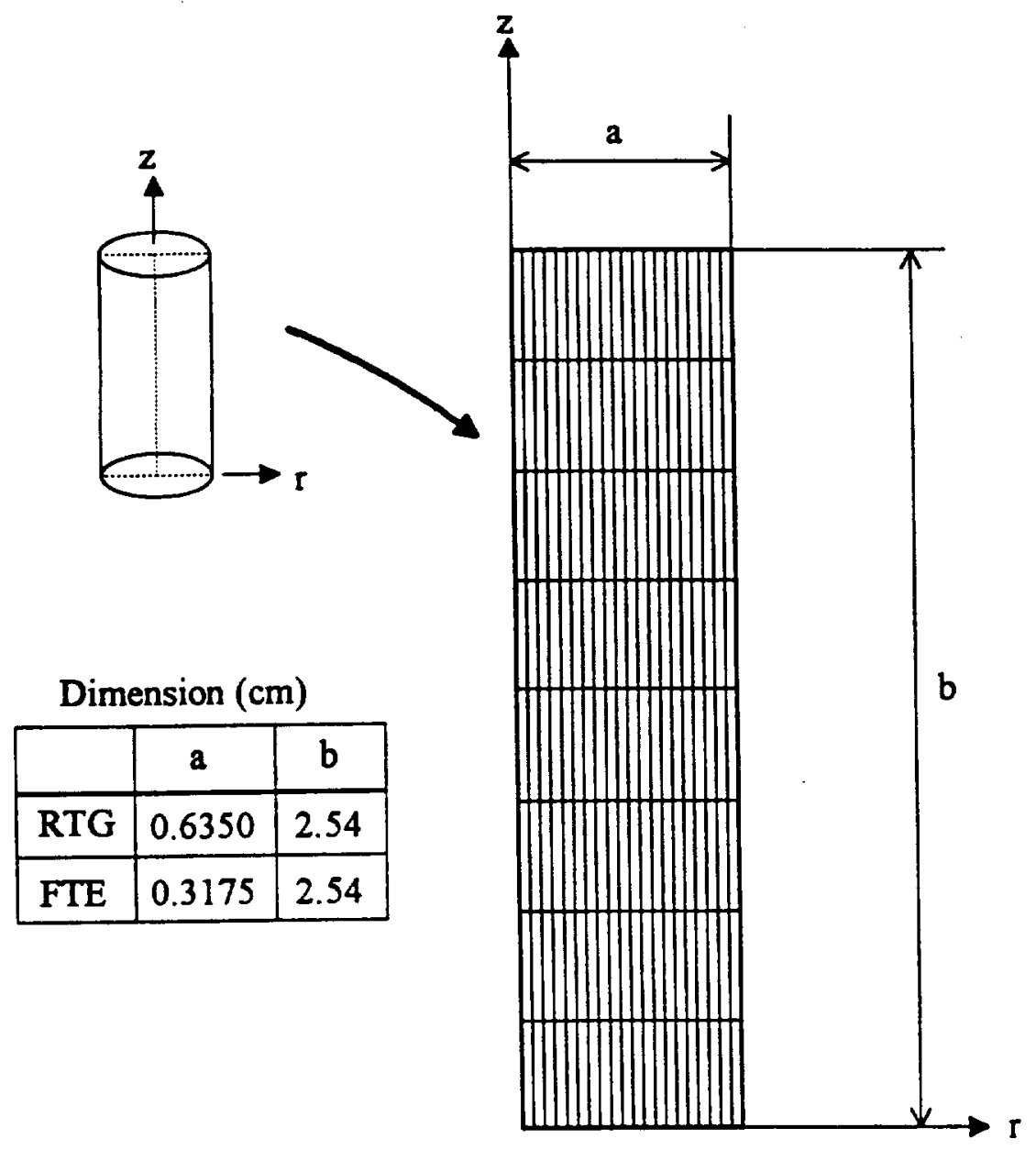




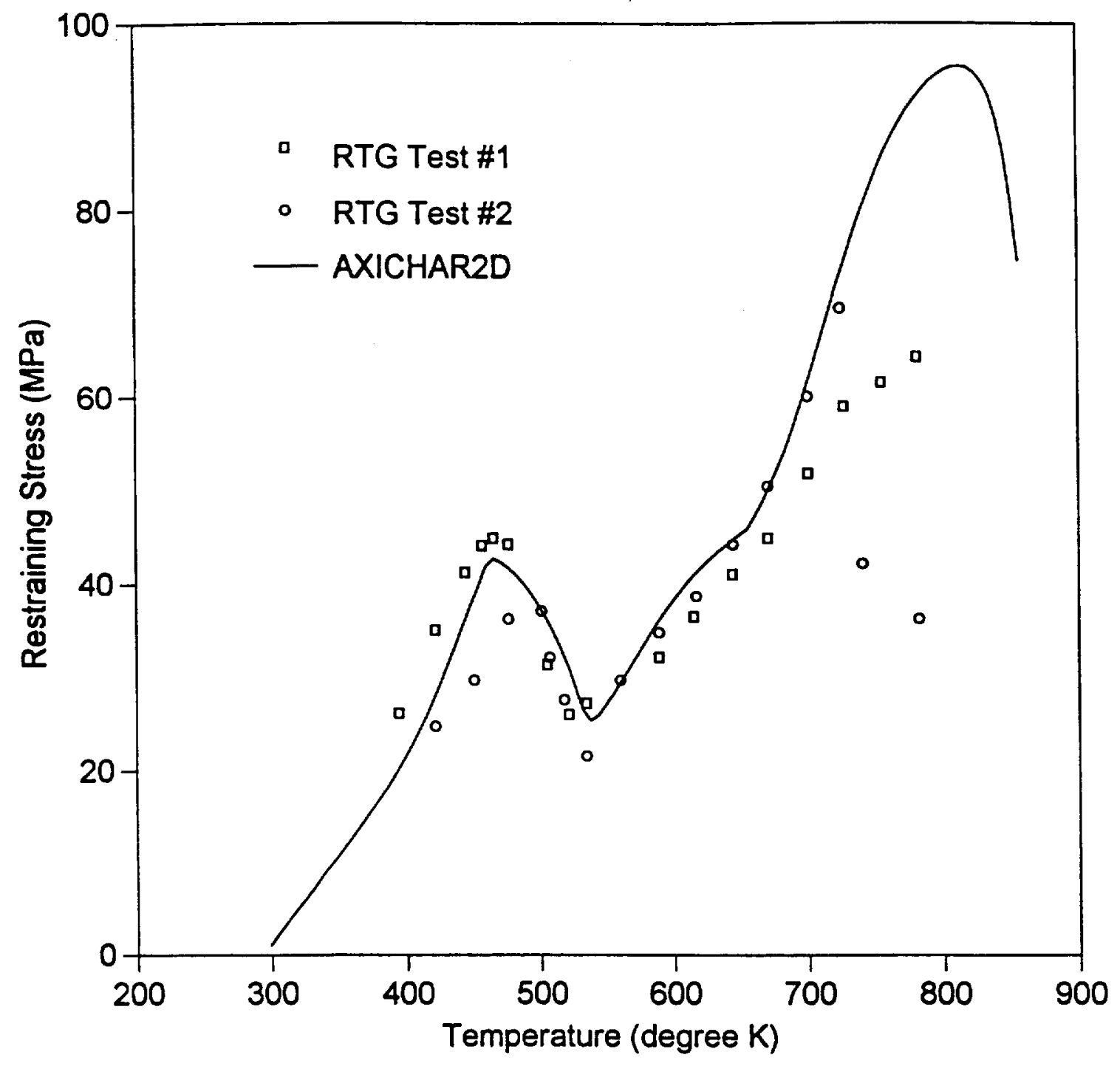




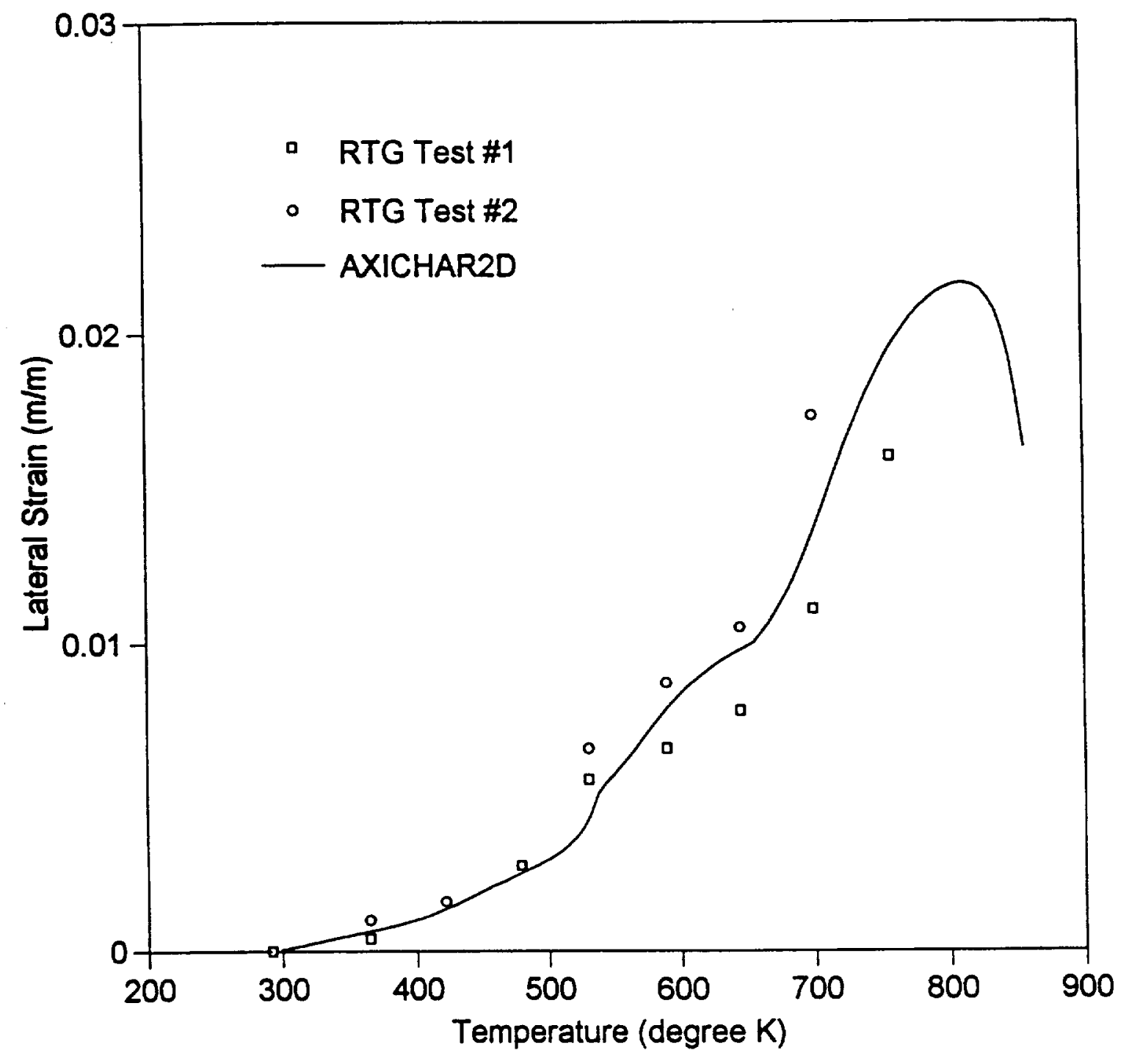




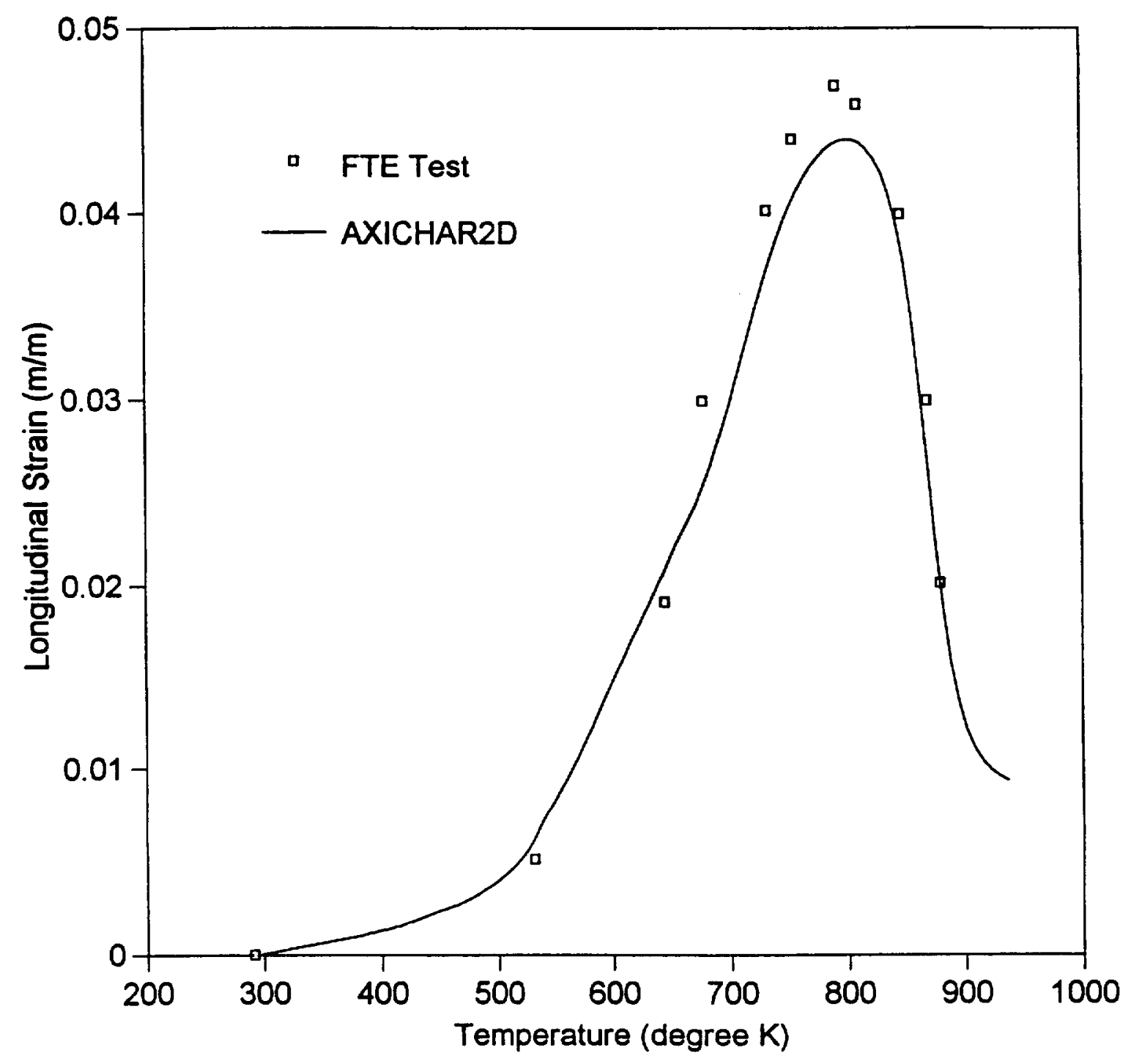




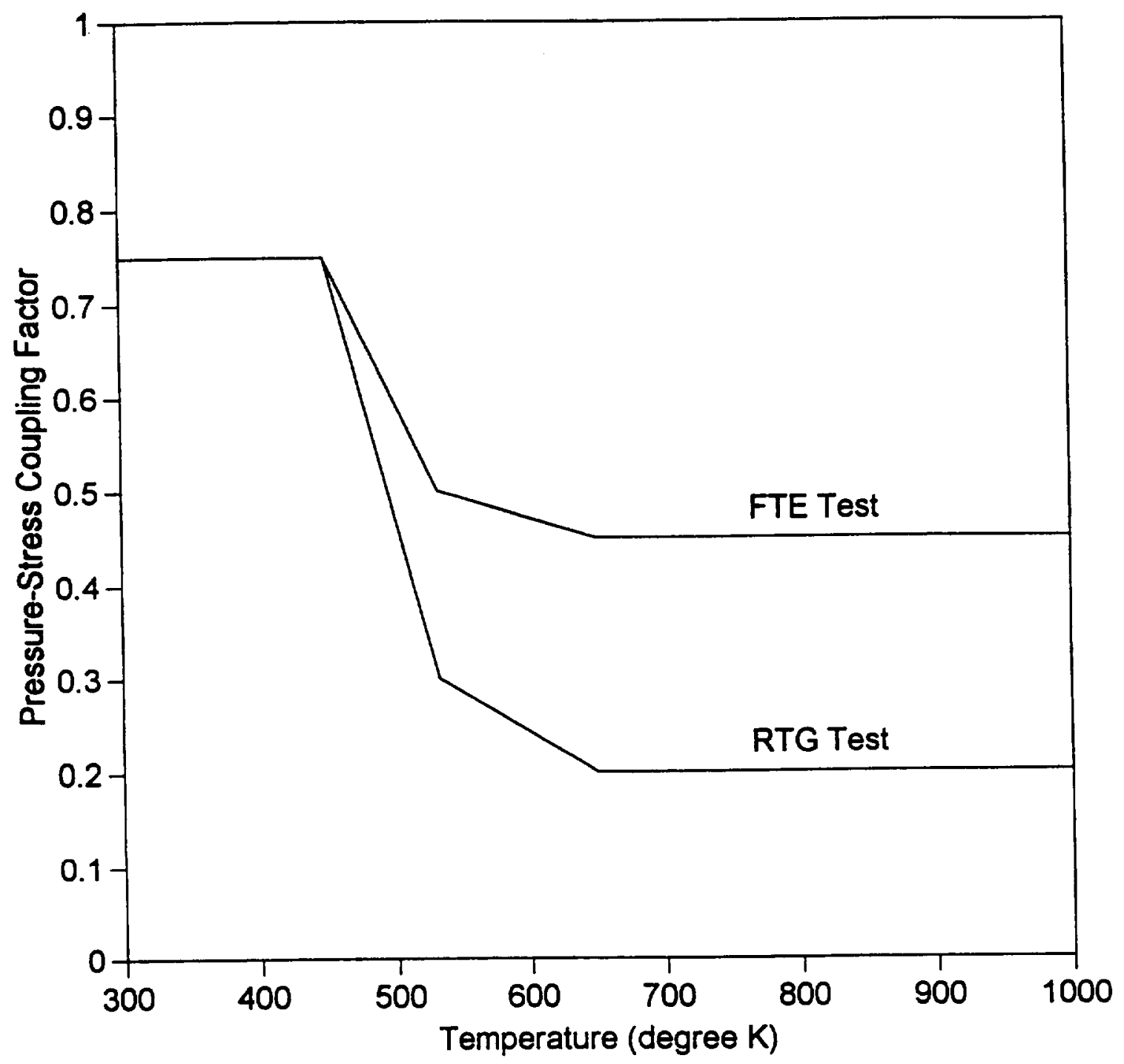

